\title{
Classical Schwinger Terms
}

\author{
DAVID G. BOULWARE ${ }^{\star}$ \\ Physics Department, University of Washington, Seattle, Washington \\ S. DESER ${ }^{\star \star}$ \\ Physics Department, Brandeis University, Waltham, Massachusetts
}

Received July 3, 1970

\begin{abstract}
Schwinger terms must be present in equal time ground state Poisson brackets among currents unless the latter are stationary in that state.
\end{abstract}

A general result in quantum mechanics is the necessary non-vanishing of the equal time commutator of any operator with its time derivative. Only the existence of a unique lowest energy state and the positivity of the Hilbert space metric enter into the derivation which uses the equation of motion

$$
\dot{A} \equiv \frac{\partial}{\partial t} A(t)=-i[A(t), H],
$$

to evaluate the vacuum expectation value ${ }^{1}$

$$
\begin{aligned}
\langle 0|[A(t), \dot{A}]| 0\rangle & =-i\langle 0|[A(t),[A(t), H]]| 0\rangle \\
& =2 i\langle 0|A(t) H A(t)| 0\rangle \\
& =2 i \sum_{n} E_{n}|\langle n|A(0)| 0\rangle|^{2} .
\end{aligned}
$$

The final summation is positive semi-definite since $E_{n}$ is; it can vanish only if the vacuum is an eigenstate of $A$. An immediate consequence in local field theory is that the equal time commutator $\left[j^{0}(r), j^{k}\left(r^{\prime}\right)\right]$ of a conserved current $j^{u}(x)$ cannot vanish $[1,2]$, since its divergence

$$
\left\langle 0\left|\left[j^{0}(\boldsymbol{r}), \nabla_{k}^{\prime} j^{k}\left(\boldsymbol{r}^{\prime}\right)\right]\right| 0\right\rangle=-\left\langle 0\left|\left[j^{0}(\boldsymbol{r}), \partial_{0} j^{0}\left(\boldsymbol{r}^{\prime}\right)\right]\right| 0\right\rangle
$$

must be non zero (and a non vanishing local current cannot annihilate the vacuum) [3]. A more precise result is that, assuming the equal time

* Supported in part by U.S.A.E.C. Contract AT(45-1)1388 B.

$\star \star$ Supported in part by U.S.A.F. OAR under OSR Grant 70-1864.

1 The zero of energy is chosen so that the vacuum lowest energy state has zero energy. The vacuum state is denoted by $|0\rangle$. 
commutator exists, then in the general expression

$$
-i\left\langle 0\left|\left[j^{0}(\boldsymbol{r}), \boldsymbol{j}(0)\right]\right| 0\right\rangle=\sum_{n=0} c_{n} \nabla\left(-\nabla^{2}\right)^{n} \delta(\boldsymbol{r}),
$$

the vanishing of $c_{0}$ implies [4] that of the operator $j^{0}$, irrespective of conservation, provided only that relativistic invariance holds.

In this note we show that the necessity of Schwinger terms is not a purely quantum phenomenon, but has a somewhat weaker classical counterpart. That Schwinger terms can exist should not be surprising: In the algebra of fields [5] for example, the current is linear in canonical variables. The commutator of Eq. (4) is then just proportional to a canonical commutation relation, and so should survive the transition ${ }^{2}$ from commutator to Poisson bracket. However, because there is no classical analogue to vacuum fluctuations it is possible for a local field to "annihilate" the vacuum, hence it is possible for the commutator to vanish. Furthermore, we will see that it is not necessary for $c_{0}$ to be non vanishing even if $j^{u}$ is not extremal at vacuum; it is only necessary that some $c_{n}$ be non zero (all must be $\geqq 0$ ).

A stable classical ground state (vacuum state) is characterized in terms of the Hamiltonian, by

and

$$
\left.\frac{\partial H}{\partial \chi^{a}}\right|_{0}=0
$$

$$
\left.\frac{\delta^{2} H}{\delta \chi^{a} \delta \chi^{b}}\right|_{0} \equiv \mathscr{H}_{a b}>0
$$

where $\chi^{a}$ represents both the canonical variables and their conjugate momenta, $q(x)^{n} \equiv \chi(x)^{2 n-1}, p(x)^{n} \equiv \chi(x)^{2 n}$. The coordinate dependence will, in general, be suppressed. The subscript zero on any quantity indicates its value in the ground state and we translate the fields $\chi$ so that $\chi=0$ there. The matrix $(\mathscr{H})_{a b}=\mathscr{H}_{a b}$ is positive definite by the ground state stability assumption.

At vacuum, the equal time Poisson bracket

$$
[A, B] \equiv \sum_{n} \frac{\partial A}{\partial q^{n}} \frac{\partial B}{\partial p^{n}}-\frac{\partial A}{\partial p^{n}} \frac{\partial B}{\partial q^{n}} \equiv \frac{\partial A}{\partial \chi} \eta \frac{\partial B}{\partial \chi}=-\frac{\delta B}{\delta \chi} \eta \frac{\delta A}{\delta \chi}
$$

combined with the role of the Hamiltonian as the generator of time translations

$$
\dot{A} \equiv \frac{\partial}{\partial t} A=[A, H]
$$

\footnotetext{
${ }^{2}$ We do not consider spinor fields.
} 
yields the result, at vacuum,

$$
\begin{aligned}
{\left.[A, \dot{A}]\right|_{0} } & =\left.[A,[A, H]]\right|_{0}=-\left.\left[A, \frac{\delta H}{\delta \chi} \eta \frac{\delta A}{\delta \chi}\right]\right|_{0} \\
& =\left(\eta \frac{\delta A}{\delta \chi}\right)_{0}^{T} \mathscr{H}\left(\eta \frac{\delta A}{\delta \chi}\right)_{0} \geqq 0
\end{aligned}
$$

and the equality can hold only if $\left.\frac{\delta A}{\delta \chi}\right)_{0}=0^{3}$. But this is just the condition that $A$ be extremal at vacuum, the classical analogue to the vacuum being an eigenstate of $A^{4}$. However, there is not classical counterpart to the necessary non vanishing of a local field, e.g., $j^{u}(x)$, applied to the vacuum; the Schwinger terms exist only if $\left(\delta j^{u} / \delta \chi\right) \neq 0$. In particular, the ordinary bilinear boson currents, whose $\chi$ derivatives vanish at vacuum, have vanishing Schwinger terms.

We will now derive from Lorentz convariance, irrespective of conservation, the more specific prediction, Eq. (4), that the equal time Poisson bracket of $j^{0}$ with $j^{k}$ must have a gradient of a delta function term. First, we will establish that $\left[j^{0}(\boldsymbol{r}), \boldsymbol{j}\left(\boldsymbol{r}^{\prime}\right)\right]_{0}$ is proportional to $\mathscr{H}$, then that $\mathscr{H}$ is proportional to $-\tilde{c}: \nabla \nabla \delta\left(\boldsymbol{r}-\boldsymbol{r}^{\prime}\right)$. The latter fact is plausible, since a general Lorentz covariant Hamiltonian is expected to involve terms $(\nabla q)^{2}$ for small excitations around vacuum. [Alternatively, such terms are expected in order that $\ddot{\chi}=[[\chi, H], H]$ contain the $\nabla^{2} \chi$ terms to combine with $\ddot{\chi}$ to form D'Alembertian.]

Consider, at $t=0$, the Poisson bracket

$$
\left[j^{0}(\boldsymbol{r}), J^{0 k}\right]=-x^{k} \partial_{0} j^{0}(\boldsymbol{r}) j^{k}(\boldsymbol{r})
$$

then take the Poisson bracket of this equation with $j^{0}\left(\boldsymbol{r}^{\prime}\right)$. The double Poisson bracket on the left is evaluated using the Jacobi Identity and Lorentz invariance of the vacuum which implies that $\left[O, J^{\mu \nu}\right]_{0}=0$ for any quantity $O^{5}$. The result of these operations, independent of whether $j^{\mu}$ is conserved, is, using $\dot{A}=[A, H]$ and the invariance of the vacuum

3 The matrix $\eta$ possesses an inverse; hence, $\eta \delta A / \delta \chi$ cannot vanish unless $\delta A / \delta \chi$ itself does. Note that the classical result requires the ground state to be a local extremum only whereas the quantum vacuum had to be an absolute minimum. There is a further analogue, for the second time derivative $[A, \ddot{A}]$ must vanish in both the quantum and classical cases.

${ }^{4}$ One way to see this is to observe that if $A \bar{\psi}=a \bar{\psi}$, then $\bar{\psi}^{+}[A, O] \bar{\psi}=0$ for all $O$; classically, $[A, O]=0$ implies $\delta A / \delta \chi=0$, hence we infer the correspondence.

5 This follows directly from the definition of "Lorentz invariance of the vacuum"; we mean that, in the vacuum no quantity changes under a Lorentz transformation, i.e.,

$$
\left[O, J^{\mu \nu}\right]=0=\frac{\partial O}{\delta \chi} \eta \frac{\delta J^{\mu \nu}}{\delta \chi} \text { or } \frac{\delta J^{\mu \nu}}{\delta \chi}=0 .
$$


under time translations,

$\left[j^{0}(\boldsymbol{r}), j^{k}\left(\boldsymbol{r}^{\prime}\right)\right]_{0}-\left[j^{0}\left(\boldsymbol{r}^{\prime}\right), j^{k}(\boldsymbol{r})\right]_{0}=\left(r-\boldsymbol{r}^{\prime}\right)^{k}\left(\eta \frac{\delta j^{0}\left(\boldsymbol{r}^{\prime}\right)}{\delta \chi}\right)^{T} H\left(\eta \frac{\delta j^{0}(\boldsymbol{r})}{\delta \chi}\right)$

If $j^{0}$ is the generator of some transformation, then $\delta j^{0}\left(\boldsymbol{r}^{\prime}\right) / \delta \chi\left(\boldsymbol{r}^{\prime \prime}\right)$ must have a $\delta\left(\boldsymbol{r}^{\prime}-\boldsymbol{r}^{\prime \prime}\right)$ term; we assume that it does (if it does not, similar arguments will apply to some $c_{n}$ in the general Poisson bracket or commutator form, Eq. (4), for $n>0{ }^{6}$. Then since $H$ must be local, it is a series of derivatives of delta functions:

$$
H=[h+\boldsymbol{\alpha} \cdot \nabla-\nabla \cdot \tilde{c} \cdot \nabla \ldots \ldots] \delta\left(\boldsymbol{r}-\boldsymbol{r}^{\prime}\right)
$$

and the factor $\left(\boldsymbol{r}-\boldsymbol{r}^{\prime}\right) H$ becomes

$$
\boldsymbol{\alpha} \delta\left(\boldsymbol{r}-\boldsymbol{r}^{\prime}\right)+2 \tilde{c} \cdot \nabla \delta\left(\boldsymbol{r}-\boldsymbol{r}^{\prime}\right)
$$

Furthermore $H$ must be a symmetric matrix, hence $\alpha$ must be antisymmetric and, comparing with Eq. (4), we find

$$
\begin{aligned}
{\left[j^{0}(\boldsymbol{r}), j^{k}\left(\boldsymbol{r}^{\prime}\right)\right] } & =\left(\eta \delta j^{0} / \delta \chi\right)^{T} \tilde{c} \cdot \nabla\left(\eta \delta j^{0} / \delta \chi\right) \\
& =c_{0} \nabla^{k} \delta\left(r-r^{\prime}\right), c_{0} \geqq 0
\end{aligned}
$$

where $c_{0}$ can vanish only if $\delta j^{0} / \delta \chi$ does or if $\tilde{c}=0$. We now show that $\tilde{c}$ cannot be zero in a Lorentz covariant theory. The structure relations of the Poincaré group are

and

$$
\left[J^{0 k}, P^{l}\right]=-\delta^{k l} P^{0} \simeq-\delta^{k l} \frac{1}{2} \chi \mathscr{H} \chi
$$

$$
\left[J^{0 k}, P^{0}\right]=-P^{k}=\frac{1}{2} \sum_{n} \int d^{3} r p^{n}(\boldsymbol{r}) \nabla^{k} q^{n}(\boldsymbol{r})=\frac{1}{2} \chi \eta \nabla^{k} \chi
$$

The Hamiltonian $P^{0}$ has been evaluated near vacuum and the momentum $P^{k}$ is exact. The fields $\chi$ must transform as some representation of the Lorentz group,

$$
\left[J^{0 k}, \chi(x)\right]=\left(x^{0} \partial^{k}-x^{k} \partial^{0}\right) \chi(x)+S^{0 k} \chi(x)
$$

For $t=0$ and near vacuum, this equation becomes

$$
\left[J^{0 k}, \chi(x)\right]=\left(x^{k}(\eta \mathscr{H})+S^{0 k}\right) \chi(x) .
$$

Now, assume that $\mathscr{H}$ has no second or higher derivatives of a delta

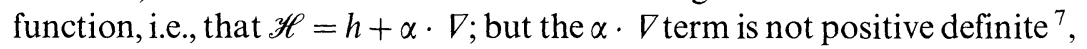
hence it must vanish and we can rewrite the structure relations, Eqs.(15),

${ }^{6}$ In the classical, as in the quantum, theory all the $c_{n}$ 's in Eq. (4) are nonnegative.

7 This conclusion is, of course, not valid for anticommuting variables; there the matrix $\mathscr{H}$ must be antisymmetric rather than symmetric, forcing instead $\chi \mathscr{H} \chi \sim \chi \alpha \cdot \nabla \chi$ with no $\nabla^{2}$ terms. 
near vacuum, as

and

$$
\left[J^{0 k}, P^{l}\right]=\chi \eta \nabla^{l}\left(x^{k} \eta h+S^{0 k}\right) \chi=-\frac{1}{2} \delta^{k l} \chi h \chi
$$

$$
\left[J^{0 k}, P^{0}\right]=\chi h\left(x^{k} \eta h+S^{0 k}\right) \chi=-\frac{1}{2} \chi \eta \nabla^{k} \chi .
$$

Only the symmetric part of the matrix between the $\chi$ 's contributes, hence the first equation reads

$$
\frac{1}{2} h=\frac{1}{2}\left[\nabla^{l} x^{k} h-h x^{k} \nabla^{l}-\nabla^{l} \eta S^{0 k}-S^{0 k^{T}} \eta \nabla^{l}\right]
$$

or, since, by locality, $\left[S^{0 k}, \nabla^{l}\right]=0$,

$$
\eta S^{0 k}+S^{0 k^{T}} \eta=0
$$

and the second equation reads

or

$$
\begin{aligned}
\frac{1}{2} \eta \nabla^{k} & =-\frac{1}{2} h S^{0 k}-\frac{1}{2} S^{0 k^{T}} h \\
& =-\frac{1}{2}\left[h S^{0 k}+\eta S^{0 k} \eta h\right] \\
& =\frac{1}{2} \eta\left[\eta h, S^{0 k}\right]
\end{aligned}
$$

$$
\nabla^{k}=\left[\eta h, S^{0 k}\right] \text {. }
$$

The matrix $\eta h$ is the direct product of a finite dimensional matrix and the unit matrix $\left\langle\boldsymbol{r}|1| \boldsymbol{r}^{\prime}\right\rangle=\delta\left(\boldsymbol{r}-\boldsymbol{r}^{\prime}\right)$, thus, only the finite dimensional factor can produce a non vanishing commutator and the trace over the finite dimensional subspace must vanish on the right and does not on the left. Hence, Eq. (21) is impossible; the only assumption (aside from Lorentz covariance) which went into this was the vanishing of $\tilde{c}$ and all higher derivative terms, and we have proven that they cannot vanish. Thus the Poisson bracket, Eq. (14), is established with a nonvanishing $c_{0}$ (or $c_{n}>0$ ).

In summary, we have shown that classical boson currents containing terms linear in the canonical variable must have Schwinger terms in their ground state Poisson brackets, just as do their commutator counterparts.

\section{References}

1. Goto, I., Imamura, T.: Prog. Theor. Phys. 14, 396 (1955).

2. Schwinger, J.: Phys. Rev. Letters 3, 296 (1959).

3. Federbush, P., Johnson, K.: Phys. Rev. 120, 1926 (1960).

4. Boulware, D. G., Deser, S.: Phys. Rev. 151, 1278 (1966).

5. Kroll, N., Lee, T. D., Zumino, B.: Phys. Rev. 157, 1376 (1967).

David G. Boulware

Physics Department

University of Washington

Seattle, Wash. 98105, USA
S. Deser

Physics Department

Brandeis University

Waltham, Mass. 02154, USA 
